\title{
Modeling of biocide action against biofilm
}

\section{Author: Tianyu Zhang}

NOTICE: The final publication is available at Springer via http://dx.doi.org/10.1007/ s11538-012-9719-z.

Zhang T, "Modeling of biocide action against biofilm," Bulletin of Mathematical Biology, February 2012 74(6):1427-1447

Made available through Montana State University's ScholarWorks

scholarworks. montana.edu 


\section{Modeling of Biocide Action Against Biofilm}

\section{Tianyu Zhang}

We consider the mathematical model of dynamic antimicrobial action against bacterial biofilms. A mixture model is used in which the biofilm consisting of live and dead bacteria is modeled as one fluid component, while the solvent contain-ing biocide is modeled as the other, and each component is represented by its volume fraction. The whole system is assumed to be an incompressible fluid and the veloc-ity is governed by the Navier-Stokes

equation. Biocide kills the live bacteria and its transport is governed by an advectionreaction-diffusion equation. Certain biocide also weakens the mechanical cohesiveness of the biofilm and results in biofilm re-moval under the shear stress of the external flow.

Spatial and temporal patterns of antimicrobial action of three different biocides are considered and numerical simula-tion results by finite difference method are presented.

Keywords Biofilm $\cdot$ Biocide $\cdot$ Mixture model $\cdot$ Finite difference method

\section{Introduction}

Biofilms are complex three-dimensional communities attached to a surface where bacterial cells are embedded in a matrix mainly composed of extracellular poly-meric substances (EPS) (Costerton et al. 1995). Biofilms have profound impact on industrial productivity and competitiveness. Their pervasive effects on water quality, power generation, energy efficiency,

human and animal health, and product quality could be extremely costly to industry and harmful to health. For example, bacterial biofilms cause severe problems in medical science including dental plaque (Gibbons and van Houte 1980), contaminated endoscopes and contact lenses (Schein et 
1989), infection of medical implants (Passerini et al. 1992), and encrustation of urethral catheters (Stickler et al. 1993). Biofilms can withstand host immune responses (Jesaitis et al. 2003) and are much more resistant to antibiotic treatments than their nonattached, individual, free-living (planktonic) counterparts (Stewart and Costerton 2001). As pointed out in Lewis (2001), Stewart (2002), some generally accepted tolerance mechanisms at work in biofilms include poor antibiotic penetration, nutrient limitation and slow growth, adaptive stress responses, and formation of persister cells. For these reasons, biofilm infections are persistent, and individuals often show recurring symptoms following antibiotic therapy. Therefore, an effective strategy for removal of biofilm is of great practical importance and tremendous interest.

Antimicrobial treatment of biofilms is a complex phenomenon involving various biological, chemical, and physical processes and mathematical modeling proves to be a powerful tool in investigating these mechanisms. There exists a variety of mathematical models describing the action of antibiotics and other disinfectants against biofilms (Anguige et al. 2005; Dodds et al. 2000; Demaret et al. 2008; Sanderson and Stewart 1997; Stewart 1996; Szomolay et al. 2005). For a more extensive review of mathematical description of biofilm disinfection, we refer to Klapper and Dockery (2010). Many of these models are one-dimensional and often limited to steady state situation, neglecting the hydrodynamic contribution to the process. There are higher-dimensional models which take account of the fluid mechanics and convective transport of antimicrobial agents (Chambless et al. 2006; Cogan 2008; Cogan et al. 2005; Eberl and Sudarsan 2008). Most of these models focus on slow flow regime (small Reynolds number) and use Stokes equation to describe the flow. For example, Eberl and Sudarsan (2008) applies a degenerate diffusion-reaction model to investigate how convective transport of nutrients and disinfectants due to bulk flow hydrodynamics affects the balance between growth and disinfection processes, where slow flow fields with Reynolds number in the range of $10^{-7} \sim 10^{-2}$ are studied and the biofilm is treated as a rigid nondeformable body. Cogan et al. (2005), Cogan (2008) present two-dimensional models for antimicrobial agents against biofilms with fluid dynamics incorporated. Biofilm is treated as a rigid body in Cogan et al. (2005) and is allowed to move as a viscous fluid (with viscosity 500 times that of water) in Cogan (2008), and Stokes equation is used in both studies. The model used in Chambless et al. (2006) contains stochastic components and requires multiple runs. Furthermore, many of the models do not account for the effect of the antimicrobial agents on the mechanical properties of the biofilms.

Although many biofilm studies fall into the low Reynolds number regime and Stokes equation describes the flow appropriately, there are certain cases where the Reynolds number is not small and full Navier-Stokes equation should be used. One particular example involves using the shear stress from the external flow as a mechanism to remove the biofilm. A recent paper by Davison et al. (2010) presents some experimental results on spatial and temporal patterns of biocide action against Staphylococcus epidermidis biofilms and underscore the distinction between biofilm removal and killing. The authors examined four antimicrobial agents: chlorine, glutaraldehyde, a quaternary ammonium compound (QAC), and an antimicrobial peptide, nisin. The behavior of these four antimicrobial agents against biofilm was investigated by visualizing the spatial and temporal patterns of the treated biofilms based on loss of 
an unbound fluorophore loaded into the cytoplasm of bacterial cells. All biocides but glutaraldehyde caused loss of membrane integrity and, therefore, killed the bacterial cells, and among those three only chlorine caused some removal of biomass from the biofilm. The experiment in Davison et al. (2010) involves a flow of Reynolds number around 20. We remark that "biocide" and "antimicrobial agent" are used interchangeably throughout the paper.

In this paper, we extend the previous mixture biofilm model developed by Zhang et al. (2008b) to qualitatively capture the experimental results in Davison et al. (2010). Efforts are made to build a mathematical model which is suitable for characterizing the biocide effect on biofilm under moderate or fast flow conditions (Reynolds number is not so small) and incorporates the effect of the biocide on the mechanical property of the biofilm. In this model, Navier-Stokes equation is used to describe the flow and the transport of biocide is mainly through convection outside the biofilm and through diffusion inside the biofilm. The killing effectiveness of the biocides is determined by many factors and here we focus on the penetration of the biocides into the biofilm through diffusion and their reaction with the biofilm. The effective diffusivity of the biocide in the biofilm is treated as a function of the biofilm volume fraction. The weakening of the mechanical property of the biofilm caused by chlorine is modeled by making the effective viscosity of the biofilm a function of both the chlorine concentration and the duration of the treatment by chlorine.

The paper is organized as follows. First, we develop the model describing the transport and reaction of the biofilm and the biocide, and the interaction between the biofilm and the external flow. Then we give a brief description of the numerical method used to simulate the model as well as the parameter values used in the computation. Finally, we present some 2D numerical results on the action of different biocides against biofilms and the comparison with the experimental results in Davison et al. (2010).

\section{Model Description}

\subsection{Overview}

The experimental setup in Davison et al. (2010) is as follows. S. epidermidis biofilms were grown in a glass capillary biofilm reactor with inside diameter $0.9 \mathrm{~mm}$ under continuous flow for 24 hours. There were three subsequent phases of staining and observation: stain, rinse, and treat. In the first phase, the biofilm was statically stained with Calcein-AM (CAM) for 1 hour. In the second phase, the biofilm was rinsed by pumping sterile phosphate-buffered saline (PBS) to remove excess CAM substrate and allow the intracellular fluorescence to stabilize. In the third phase, an antimicrobial agent was pumped through the capillary at the flow rate of $1 \mathrm{ml} \mathrm{min}^{-1}$ for 1 hour and the spatiotemporal patterns of loss of fluorescence were observed with the confocal scanning laser microscope (CSLM).

In this paper, we focus on the modeling of the third phase of the experiment (the treatment by biocide) and assume the growth of the biofilm during this phase is negligible. The no growth assumption is appropriate since there is no substrate available 
during the disinfection phase after rinsing and the time scale (1 hour) is sufficient fast comparing to the typical time scale (days) for the growth of a biofilm. On the other hand, the time scale of the disinfection phase of the experiment is sufficiently slow comparing to the relaxation time of biofilm (on the order of seconds), and biofilm can in fact be well approximated as a viscous fluid (Shaw et al. 2004) with viscosity much larger than that of the solvent. In our model, we assume the biofilm consists of live and dead bacteria cells, and the extracellular polymeric substance (EPS) of the biofilm is not modeled explicitly. The biocide is dissolved in the solvent and is brought into the biofilm-occupied region by the external flow with a constant biocide concentration. Biocide penetrates the biofilm mainly by diffusion, and the diffusion coefficients of the biocides are often largely reduced in the biofilm probably due to sorption and/or reaction of the biocide in the biofilm. As a consequence, the killing effectiveness of the biocide is largely retarded in the biofilm. However, experimental results from Davison et al. (2010) show exception such as nisin, whose killing effectiveness in the biofilm was not reduced as much as other biocides, and the explanation in Davison et al. (2010) is that nisin is not neutralized or bounded by the cells or matrix of the biofilm. Chlorine was the only antimicrobial agent that caused removal of biomass from the biofilm. The video from the experiment (supplemental material of Davison et al. 2010) showed that the surface layer of the biofilm was fluidized by chlorine and behaved as a viscous fluid. When the liquefied biomass reached the downstream edge of the biofilm, it could be stripped from the biofilm and carried away by the flow.

\subsection{Governing Equations}

Following Zhang et al. (2008b), we adopt the mixture model in describing the biocide action against biofilm in a two-dimensional rectangular domain. One fluid component is the biomaterial which consists of live and dead bacteria cells with volume fraction $\phi_{b l}$ and $\phi_{b d}$ respectively, and the total biomaterial volume fraction is $\phi_{b}=\phi_{b l}+\phi_{b d}$. The other fluid component is the solvent with volume fraction $\phi_{s}$ which contains the dissolved biocide. Due to the constraint $\phi_{s}+\phi_{b}=\phi_{s}+\phi_{b l}+\phi_{b d}=1$, only two volume fractions $\phi_{b l}$ and $\phi_{b d}$ need to be computed. We remark that $\phi_{s}, \phi_{b l}$, and $\phi_{b d}$ are dimensionless since they are volume fractions, and average quantities (density, viscosity) using them as weight still have the their original units. These two components are modeled as viscous fluids with different viscosities, and the whole system is assumed to be an incompressible fluid whose average velocity $\mathbf{v}$ is governed by the Navier-Stokes equation. The antimicrobial agent concentration is denoted by $d$.

The reaction-diffusion-advection equations for $d, \phi_{b l}$, and $\phi_{b d}$ are

$$
\begin{aligned}
& \frac{\partial}{\partial t}\left(\phi_{s} d\right)+\nabla \cdot\left(\phi_{s} \mathbf{v} d-D_{e} \phi_{s} \nabla d\right)=-\phi_{b l} \frac{K_{1} d}{d_{0}+d}, \\
& \frac{\partial \phi_{b l}}{\partial t}+\nabla \cdot\left(\phi_{b l} \mathbf{v}\right)=\nabla \cdot\left(\Lambda \phi_{b l} \nabla \frac{\delta f}{\delta \phi_{b l}}\right)-\phi_{b l} \frac{K_{2} d}{K_{d}+d}, \\
& \frac{\partial \phi_{b d}}{\partial t}+\nabla \cdot\left(\phi_{b d} \mathbf{v}\right)=\nabla \cdot\left(\Lambda \phi_{b d} \nabla \frac{\delta f}{\delta \phi_{b d}}\right)+\phi_{b l} \frac{K_{2} d}{K_{d}+d} .
\end{aligned}
$$


The biocide concentration $d$ is multiplied by $\phi_{s}$ in its transport equation since it is dissolved in and carried by the solvent. $D_{e}$ is the effective diffusion coefficient of the biocide in the biofilm and it may depend on the biomaterial volume fraction $\phi_{b}$. We assume that the biocide only reacts with the live bacteria cells. Its decay rate is modeled by Monod kinetics with maximum decay rate $K_{1}$ and Monod half-saturation coefficient $d_{0}$, and is also proportional to live bacteria volume fraction $\phi_{b l}$.

The decay rate of the live bacteria cells $\left(\phi_{b l}\right)$ due to the biocide action is also modeled by Monod kinetics with maximum decay rate $K_{2}$ and Monod half-saturation coefficient $K_{d}$, and is proportional to $\phi_{b l}$. The growth rate of the dead bacteria cells $\left(\phi_{b d}\right)$ is exactly the negative of the decay rate of $\phi_{b l}$, reflecting that live bacteria are converted to dead bacteria due to biocide action. We remark that many antimicrobial agents are most effective at killing respiring bacteria (Costerton et al. 1995), and the decay rate of live bacteria generally will depend on the nutrient concentration as well. However, we do not include this factor in our model since the biofilm was rinsed and there was no nutrient in the antimicrobial agent treatment phase in the experiment described above. This factor can be readily accounted for by introducing a new variable for the nutrient concentration which is governed by an equation similar to that of $d$, and modifying the reaction terms for $\phi_{b l}$ and $\phi_{b d}$ accordingly. For the intermixing of the small molecule solvent with the large molecule biofilm, we adopt the extended Flory-Huggins chemical free energy density $f$ from Doi (1995) defined by

$$
f\left(\phi_{b}\right)=k_{B} T\left[\frac{\Gamma_{1}}{2}\left\|\nabla \phi_{b}\right\|^{2}+\Gamma_{2}\left(\frac{1}{N} \phi_{b} \ln \phi_{b}+\left(1-\phi_{b}\right) \ln \left(1-\phi_{b}\right)+\chi \phi_{b}\left(1-\phi_{b}\right)\right)\right],
$$

which consists of mixing energy, transitional energy and mixing entropy components. Here, $k_{B}$ is the Boltzmann constant and $T$ is temperature, $\Gamma_{1}$ and $\Gamma_{2}$ are the distortional and bulk mixing energy strength, respectively, $N$ is the generalized polymerization parameter of the biofilm, and $\chi$ is the Flory-Huggins mixing parameter. The variation of $f$ with respect to $\phi_{b}$ (known as the chemical potential) is given by

$$
\frac{\delta f}{\delta \phi_{b}}=k_{B} T\left[-\Gamma_{1} \Delta \phi_{b}+\Gamma_{2}\left(\frac{1}{N}\left(1+\ln \phi_{b}\right)-\ln \left(1-\phi_{b}\right)-1+\chi\left(1-2 \phi_{b}\right)\right)\right] .
$$

We assume the mixing properties of the live and dead bacteria cells with the solvent are the same, thus $f$ is a function of the total biomaterial volume fraction $\phi_{b}$ only. From $f\left(\phi_{b}\right)=f\left(\phi_{b l}+\phi_{b d}\right)$, the chain rule implies that $\frac{\delta f}{\delta \phi_{b}}=\frac{\delta f}{\delta \phi_{b l}}=\frac{\delta f}{\delta \phi_{b d}}$. The $f$ related terms in the equations for $\phi_{b l}$ and $\phi_{b d}$ describe the mixing flux due to the chemical potential gradient $\nabla \frac{\delta f}{\delta \phi_{b}}$, where $\Lambda$ is the biomaterial mobility parameter. Detailed discussion of the parameters in (4) as well as the mobility parameter $\Lambda$ can be found in Zhang et al. (2008a, 2008b). These extra parameters do not play an important role in the results presented here. We note that the governing equation of the total biomaterial $\phi_{b}$ is obtained by adding (2) and (3), namely,

$$
\frac{\partial \phi_{b}}{\partial t}+\nabla \cdot\left(\phi_{b} \mathbf{v}\right)=\nabla \cdot\left(\Lambda \phi_{b} \nabla \frac{\delta f}{\delta \phi_{b}}\right) .
$$


Table 1 Antimicrobial agents and their properties. Reprinted by permission of American Society for Microbiology from Davison et al. (2010)

\begin{tabular}{lrrr}
\hline Agent & MW & $D_{\text {aq }}{ }^{\mathrm{a}}\left(\mu \mathrm{m}^{2} \mathrm{~s}^{-1}\right)$ & $t_{50}(\mathrm{~s})$ \\
\hline Hypochlorous acid & 50 & 1,900 & 3 \\
Glutaraldehyde & 100 & 930 & 11 \\
QAC & 357 & 427 & 24 \\
Nisin & 3,354 & 190 & 55 \\
\hline
\end{tabular}

${ }^{\mathrm{a}} D_{\mathrm{aq}}$, diffusion coefficient in water

The whole system is modeled as an incompressible fluid, and its velocity $\mathbf{v}$ and pressure $p$ are determined by the following modified Navier-Stokes equations

$$
\begin{aligned}
& \nabla \cdot \mathbf{v}=0, \\
& \rho\left(\frac{\partial \mathbf{v}}{\partial t}+\mathbf{v} \cdot \nabla \mathbf{v}\right)=\nabla \cdot(\nu \mathbf{D})-\nabla p+\frac{\delta f}{\delta \phi_{b}} \nabla \phi_{b},
\end{aligned}
$$

with

$$
v=v\left(\phi_{b d}, \phi_{b l}, \phi_{s}, v_{b}, v_{s}, d\right), \quad \rho=\phi_{b} \rho_{b}+\phi_{s} \rho_{s}, \quad \mathbf{D}=\frac{1}{2}\left(\nabla \mathbf{v}+\nabla \mathbf{v}^{T}\right),
$$

where $v_{b}, v_{s}$ are viscosities of the untreated biomaterial and solvent, and $\rho_{b}, \rho_{s}$ are their respective densities. We approximate the biomaterial component as a viscous fluid with viscosity much larger than that of the solvent $\left(v_{b} \gg v_{s}\right)$. Note that for sufficiently slow flow time scales (as is the case here), biofilm can in fact be well approximated as a viscous fluid (Shaw et al. 2004). The effective viscosity $v$ of the whole system depends on the viscosity and volume fraction of each component of the fluid (biomaterial and solvent), and also on the biocide concentration if the biocide (such as chlorine) weakens the mechanical property of the biofilm. The additional stress terms (the ones with $f$ dependence) are forces arising from the chemical free energy; see Zhang et al. (2008b), Zhang and Klapper (2010).

\subsection{Effective Diffusion Coefficient of Biocide and Reaction with Biofilm}

The diffusivity of chemical species in biofilms could vary from 10 to 90 percent of the corresponding value in water (Characklis and Marshall 1990), depending on the types of chemical species and biofilms. In Davison et al. (2010), the biofilm diffusive penetration time $t_{50}$, which is the time required for the biocide concentration at the center of the biofilm to reach $50 \%$ of its value in the bulk fluid after the onset of biocide exposure, in the absence of reaction, was estimated for all biocides used in the experiment. Here, the formula $t_{50}=0.139 R^{2} / D_{e}$ (see Davison et al. 2010; Stewart 2003) was used, where the typical biofilm cluster radius $R$ was taken as $150 \mu \mathrm{m}$, and the effective diffusion coefficient $D_{e}$ in the biofilm was taken as $50 \%$ of the value in water for chlorine and $30 \%$ of the value in water for the other agents. Results shown in Table 1 indicate that $t_{50}$ for all biocides are in the order of seconds, and 
Table 2 Summary of effects of antimicrobial agents on S. epidemudis biofilms. Reprinted by permission of American Society for Microbiology from Davison et al. (2010)

\begin{tabular}{llllll}
\hline Agent & $C_{0}{ }^{\mathrm{a}}\left(\mathrm{mg} \mathrm{liter}^{-1}\right)$ & $T_{50 B}(\mathrm{~min})$ & $A_{60} / A_{0}$ & $I_{60} / I_{0}$ & $n^{\mathrm{b}}$ \\
\hline Control & $\mathrm{NA}^{\mathrm{c}}$ & $\mathrm{ND}^{\mathrm{d}}$ & $1.003 \pm 0.005$ & $0.954 \pm 0.031$ & 5 \\
Hypochlorous acid & 10 & $46 \pm 33$ & $0.574 \pm 0.348$ & $0.281 \pm 0.473$ & 3 \\
Hypochlorous acid & 50 & $21 \pm 16$ & $0.096 \pm 0.142$ & $0.006 \pm 0.015$ & 6 \\
Glutaraldehyde & 50 & $\mathrm{ND}$ & $1.009 \pm 0.020$ & $0.886 \pm 0.103$ & 5 \\
QAC & 50 & $25 \pm 15$ & $0.908 \pm 0.096$ & $0.030 \pm 0.014$ & 4 \\
Nisin & 50 & $4 \pm 1$ & $0.919 \pm 0.092$ & $0.006 \pm 0.001$ & 2 \\
\hline
\end{tabular}

${ }^{\mathrm{a}} C_{0}$, applied concentration

$\mathrm{b}_{n, \text { number of experiments }}$

${ }^{\mathrm{c}} \mathrm{NA}$, not applicable

${ }^{\mathrm{d}} \mathrm{ND}$, not determined, as fluorescence intensity did not reach $50 \%$ of initial intensity within the 60 -min experimental test

Table 3 Dependence of effective diffusivity $D_{e}$ on biofilm volume fraction $\phi_{b}$

\begin{tabular}{llllllll}
\hline Biofilm volume fraction $\phi_{b}$ & 0 & 0.01 & 0.025 & 0.05 & 0.1 & 0.2 & 0.4 \\
\hline Effective diffusivity $D_{e}$ & $D_{d}^{0}$ & $0.771 D_{d}^{0}$ & $0.571 D_{d}^{0}$ & $0.396 D_{d}^{0}$ & $0.241 D_{d}^{0}$ & $0.130 D_{d}^{0}$ & $0.062 D_{d}^{0}$ \\
\hline
\end{tabular}

nisin has the largest $t_{50}$ value since it has the largest molecular weight and smallest diffusion coefficient in water. However, the experimentally measured value of $T_{50 B}$, which is the time required for the fluorescence intensity at the center of a biofilm cluster to fall to one-half of its initial value after the onset of biocide exposure, was generally much larger than $t_{50}$ and in the order of minutes, as shown in Table 2. This large discrepancy suggests that sorption or reaction of biocide in the biofilm retards its penetration process. In our model equation (1)-(3), the killing rate of live bacteria cells is proportional to the biocide concentration, and the transport of biocide in biofilm is mainly due to diffusion (the advection effect is small in the biofilm since the flow velocity in the biofilm is very small due to its large viscosity). Therefore, the effective diffusion coefficient in the biofilm $D_{e}$ in (1) should account for both the diffusive penetration and action time of the biocide, and is modeled as follows. We adopt the model of $D_{e}$ by Hinson and Kocher (1996) inspired by biofilm kinetics which has been fit to experimental data. In this model,

$$
D_{e}=\frac{2 D_{d}^{0}\left(1-\tilde{\phi}_{b}\right)}{\left(2+\tilde{\phi}_{b}\right)\left(\phi_{s}+\tilde{\phi}_{n} / D_{p r}\right)},
$$

where $\phi_{s}, \tilde{\phi}_{b}$ and $\tilde{\phi}_{n}$ are the volume fractions of solvent, bacteria and EPS polymer network. We note that in the above formula for $D_{e}$, the bacteria and EPS are treated as separate components of the biofilm, and the volume fraction of the total biomaterial is $\phi_{b}=\tilde{\phi}_{b}+\tilde{\phi}_{n}$. Since our model does not account for EPS explicitly, we assume $\tilde{\phi}_{n}=$ $0.6 \phi_{b}$ and $\tilde{\phi}_{b}=0.4 \phi_{b}$ in our computation. Here, $D_{p r}$ is an empirical parameter that 
must be fit by experimental observation, and $D_{p r}=0.02$ is used in our computation following (Hinson and Kocher 1996). Table 3 gives the dependence of $D_{e}$ on $\phi_{b}$ according to (7). We note as the biofilm volume fraction $\phi_{b}$ increases from 0 to 0.4 , the effective diffusivity $D_{e}$ drops from its default value $D_{d}^{0}$ to about 6 percent of $D_{d}^{0}$.

We note that nisin has the smallest difference between $t_{50}=55$ seconds and $t_{50 B} \approx 4$ minutes. Results in Davison et al. (2010) show that nisin (molecular weight [MW], 3,354) accessed the interior of biofilm cell clusters faster than the smaller QAC (MW, 357) and chlorine (MW, 50), and killed bacteria uniformly from all parts of the biofilm. Quicker penetration for the larger agent is exactly opposite of what intuition suggests and is explained in Davison et al. (2010) as follows. It underscores the importance of the reactivity of the biocide in the biofilm in controlling its penetration, and the fast penetration of nisin indicates that it is not neutralized by the cells or matrix of the biofilm. In contrast, both QAC and chlorine must react with or be absorbed into the biofilm, interactions that retard the penetration. In our model, this fact is characterized by using a much smaller value of $K_{1}$ (the decay rate of the biocide due to reaction with the biofilm) for nisin than that for QAC and chlorine.

\subsection{Effective Viscosity of the Biomaterial}

In our model, the biofilm is treated as a viscous fluid and its mechanical strength is characterized by its viscosity. The effective viscosity $v$ of the whole system (including biomaterial and solvent) is modeled as follows. If the biocide does not weaken the mechanical property of the biofilm (such as QAC and nisin), then $v$ is the volume average of the viscosity of the untreated biomaterial $v_{b}$ and that of the solvent $v_{s}$ $\left(v_{b} \gg v_{s}\right)$, namely,

$$
v=\phi_{b} v_{b}+\phi_{s} v_{s}
$$

If the biocide weakens the mechanic property of the biofilm (such as chlorine), then

$$
v=\left[(1-\tanh (\eta)) v_{b}+\tanh (\eta) v_{s} \phi_{b d}+v_{b} \phi_{b l}+v_{s} \phi_{s}\right.
$$

where

$$
\eta(x, t)=\frac{{ }_{0}^{t} d(x, s) d s}{\bar{d} \bar{T}} .
$$

Here, $\eta$ is defined as the integral of the biocide concentration over time and normalized by the product of some reference concentration $\bar{d}$ and reference time scale $\bar{T}$, and it is a dimensionless quantity describing the accumulation of biocide over time which accounts for the time effect of biocide treatment. Equation (9) still defines $v$ by the volume average, but the viscosity of the dead bacteria is now $(1-\tanh (\eta)) v_{b}+\tanh (\eta) v_{s}$. If there is no biocide, $d=\eta=0$ and $\tanh (\eta)=0$, then $v=v_{b}\left(\phi_{b d}+\phi_{b l}\right)+v_{s} \phi_{s}=v_{b} \phi_{b}+v_{s} \phi_{s}$ which coincides with (8). If there is sufficient biocide and the treatment time is long enough, then $\eta \gg 1, \tanh (\eta) \approx 1$ and $v \approx v_{b} \phi_{b l}+v_{s}\left(\phi_{b d}+\phi_{s}\right)$. Namely, the dead bacteria cells are liquefied. Equation (10) is motivated by the following observation from Davison et al. (2010). The killed biomass is liquefied under treatment of certain biocide such as chlorine (either at high 
concentration of $50 \mathrm{ml} \mathrm{liter}{ }^{-1}$ or at low concentration of $10 \mathrm{ml} \mathrm{liter}^{-1}$ ) and its viscosity is decreased close to that of the solvent $v_{s}$, and the low concentration treatment takes longer time to liquefy the biomass. Therefore, the effective viscosity $v$ should have the following property: $v \approx v_{b} \phi_{b}+v_{s} \phi_{s}$ if $d=0$ or $t \approx 0$ (no biocide or treatment time is too short), and $v \approx v_{b} \phi_{b l}+v_{s}\left(\phi_{b d}+\phi_{s}\right)$ if $d \geq C$ and $t \gg 1$ (sufficient long treatment with enough biocide, $C>0$ is a constant). Combination of (9) and (10) satisfies this property. On the other hand, if $\eta$ in (9) only depends on the pointwise value of $d(x, t)$ itself (e.g., $\eta(x, t)=d(x, t) / \bar{d})$, since $v_{b} \gg v_{s}$ (by several orders of magnitudes), $v \approx v_{b} \phi_{b l}+v_{s}\left(\phi_{b d}+\phi_{s}\right)$ can only occur when $d \gg \bar{d}$, which implies a treatment by low biocide concentration $(d \leq \bar{d})$ will never liquefy the biomass regardless of the duration of the treatment, and conflicts the experimental results from Davison et al. (2010).

\section{Numerical Methods}

The governing equations (1)-(3) and (5)-(6) are nondimensionalized by using the scalings

$$
\tilde{\mathbf{x}}=\frac{\mathbf{x}}{h_{0}}, \quad \tilde{t}=\frac{t}{t_{0}}, \quad \tilde{\mathbf{v}}=\frac{\mathbf{v} t_{0}}{h_{0}}, \quad \tilde{p}=\frac{p t_{0}^{2}}{\rho_{0} h_{0}^{2}}, \quad \tilde{d}=\frac{d}{d_{0}},
$$

where $h_{0}$ and $t_{0}$ are the characteristic length and time scale, the characteristic biocide concentration $d_{0}$ is taken the same as the half saturation constant $d_{0}$ in the Monod kinetics, and the characteristic density $\rho_{0}$ is taken as the density of water. The dimensionless equations are solved by a finite difference method on a uniform rectangular mesh in space ( $\Delta x=\Delta y=1 / 128)$, where the dimensionless computation domain is a unit square $\Omega=[0,1] \times[0,1]$. The time step size is $\Delta t=0.5 \Delta x$ which satisfies the CFL condition since the dimensionless velocity has a maximum value 1 . Computations are checked by comparison with results from doubling of the spatial resolution to ensure that the grid is adequately fine.

Below is a brief description of the solution process and details about the numerical scheme can be found in Lindley et al. (2011), Zhang et al. (2008b). For a generic function $g(x, t)$, its numerical approximation at time $t_{n}=n \Delta t$ is denoted by $g^{n}$ and its second order extrapolation in time is denoted by $\bar{g}^{n+1}=2 g^{n}-g^{n-1}$. After numerical solutions of all unknown functions at time $t_{n}$ are obtained, iteration to the next time step $t_{n+1}$ goes in three steps as follows. In step one, the coupled continuity equation (5) and momentum transport equation (6) are solved by a velocity-corrected projection scheme (Guermond et al. 2006) to obtain the new velocity $\mathbf{v}^{n+1}$. We note that (6) depends on the bacteria volume fractions through both the effective viscosity and the chemical free energy stress term. Since their values at $t_{n+1}$ are unknown at the moment, the corresponding extrapolated values are used. In step two, the advectionreaction-diffusion equations (2)-(3) for the bacteria volume fractions $\phi_{b l}$ and $\phi_{b d}$ are solved by a Crank-Nicolson scheme with the advection term treated by a fully implicit upwind scheme, and the new values $\phi_{b l}^{n+1}, \phi_{b d}^{n+1}$ are obtained. Again, extrapolated value $\bar{d}^{n+1}$ of the biocide concentration at $t_{n+1}$ is used in (2)-(3). In step 3, 
Table 4 Parameter values used in simulations, see Sect. 3 for discussions

\begin{tabular}{|c|c|c|c|}
\hline Symbol & Parameter & Value & Unit \\
\hline$T$ & Temperature & 296 & Kelvin \\
\hline$\Gamma_{1}$ & Distortional energy & $8 \times 10^{6}$ & $\mathrm{~kg} \mathrm{~ms} \mathrm{~s}^{-2}$ \\
\hline$\Gamma_{2}$ & Strength of the bulk mixing energy & $3 \times 10^{17}$ & $\mathrm{~kg} \mathrm{~ms} \mathrm{~s}^{-2}$ \\
\hline$\chi$ & Flory-Huggins mixing parameter & 0.55 & dimensionless \\
\hline$N$ & Generalized polymerization parameter & $1 \times 10^{3}$ & dimensionless \\
\hline$\Lambda$ & Mobility parameter & $1 \times 10^{-9}$ & $\mathrm{~kg}^{-1} \mathrm{~m}^{3} \mathrm{~s}$ \\
\hline$v_{b}$ & Viscosity of the bacteria & 5 & $\mathrm{~kg} \mathrm{~m}^{-1} \mathrm{~s}^{-1}$ \\
\hline$v_{S}$ & Viscosity of the solvent & $1.002 \times 10^{-3}$ & $\mathrm{~kg} \mathrm{~m}^{-1} \mathrm{~s}^{-1}$ \\
\hline$\rho_{b}$ & Biofilm density & $1 \times 10^{3}$ & $\mathrm{~kg} \mathrm{~m}^{-3}$ \\
\hline$\rho_{s}$ & Solvent density & $1 \times 10^{3}$ & $\mathrm{~kg} \mathrm{~m}^{-3}$ \\
\hline$D_{C}^{0}$ & Aqueous diffusion coefficient of chlorine & $1.9 \times 10^{-9}$ & $\mathrm{~m}^{2} \mathrm{~s}^{-1}$ \\
\hline$D_{Q}^{0}$ & Aqueous diffusion coefficient of QAC & $0.427 \times 10^{-9}$ & $\mathrm{~m}^{2} \mathrm{~s}^{-1}$ \\
\hline$D_{N}^{0}$ & Aqueous diffusion coefficient of Nisin & $0.19 \times 10^{-9}$ & $\mathrm{~m}^{2} \mathrm{~s}^{-1}$ \\
\hline$K_{1}$ & Maximum biocide consumption rate for chlorine and QAC & $2 \times 10^{-4}$ & $\mathrm{~kg} \mathrm{~m}^{-3} \mathrm{~s}^{-1}$ \\
\hline$K_{1}$ & Maximum biocide consumption rate for nisin & $2 \times 10^{-7}$ & $\mathrm{~kg} \mathrm{~m}^{-3} \mathrm{~s}^{-1}$ \\
\hline$d_{0}$ & Biocide Monod half-saturation coefficient & $1 \times 10^{-3}$ & $\mathrm{~kg} \mathrm{~m}^{-3}$ \\
\hline$K_{2}$ & Maximum decay rate of live bacteria (due to biocide action) & $2 \times 10^{-5}$ & $\mathrm{~s}^{-1}$ \\
\hline$K_{d}$ & Live bacteria decay Monod half-saturation coefficient & $1 \times 10^{-5}$ & $\mathrm{~kg} \mathrm{~m}^{-3}$ \\
\hline $\bar{d}$ & Reference biocide concentration for weakening biomaterial & $5 \times 10^{-3}$ & $\mathrm{~kg} \mathrm{~m}^{-3}$ \\
\hline $\bar{T}$ & Reference time scale for weakening biomaterial & 120 & $\mathrm{~s}$ \\
\hline$h_{0}$ & Characteristic length scale & $10^{-3}$ & $\mathrm{~m}$ \\
\hline$t_{0}$ & Characteristic time scale & $5 \times 10^{-2}$ & $\mathrm{~s}$ \\
\hline$\rho_{0}$ & Characteristic density scale & $1 \times 10^{3}$ & $\mathrm{~kg} \mathrm{~m}^{-3}$ \\
\hline
\end{tabular}

the advection-reaction-diffusion equation (1) for biocide concentration $d$ is solved using the same numerical scheme as in step two, and the new value $d^{n+1}$ is obtained. We note the solvent volume fraction at $t_{n+1}$ is known after step two by $\phi_{s}^{n+1}=$ $1-\phi_{b l}^{n+1}-\phi_{b d}^{n+1}$, thus $d^{n+1}$ is the only unknown in (1). The resulting system of algebraic equations are solved by a GMRES iterative method (Saad and Schultz 1986).

Important parameter values used in simulations are listed in Table 4. The parameters in the chemical free energy density $f$ are taken from Zhang et al. (2008b), and the aqueous diffusion coefficients of different biocides are taken from Davison et al. (2010). The density of the biofilm is assumed to be the same as the solvent, while the viscosity of the untreated biofilm is taken to be 5000 times that of the solvent (experiments in Davison et al. 2010 show that the biomass velocity was of order $1 \mu \mathrm{m} / \mathrm{s}$, and the average bulk fluid velocity was $2 \mathrm{~cm} / \mathrm{s}$.) Due to the lack of experimentally measured results, heuristic values of the parameters in the Monod kinetic reaction between the biocide and bacteria, as well as the reference biocide concentration $\bar{d}$ and time scale $\bar{T}$ for the weakening of the biofilm, are taken such that the numerical simulation with these values qualitatively matches the experimental data. We remark 
that the biocide Monod half-saturation constant $d_{0}=1 \times 10^{-3} \mathrm{~kg} \mathrm{~m}^{-3}$ is the same as the value used in Chambless et al. (2006), while the maximum biocide consumption rate for QAC and chlorine $K_{1}=2 \times 10^{-4} \mathrm{~kg} \mathrm{~m}^{-3} \mathrm{~s}^{-1}$ is about 36 times bigger than that of the value used in Chambless et al. (2006) $\left(5.5 \times 10^{-6} \mathrm{~kg} \mathrm{~m}^{-3} \mathrm{~s}-1\right)$. On the other hand, the simulation in Chambless et al. (2006) was done for 50 hours comparing to 1 hour in our case, thus the difference in the value of $K_{1}$ is reasonable.

\section{Results and Discussion}

We consider a two-dimensional system with a square domain of size $1 \mathrm{~mm}$ by $1 \mathrm{~mm}$ (corresponding to the cross section of the glass capillary in the experimental setup in Davison et al. 2010). The initial condition is a semicircular biofilm colony consisting of only live bacteria with radius $R=0.3 \mathrm{~mm}$ and volume fraction $\phi_{b l}=0.4$ attached to the bottom of the domain. An external driven flow (parabolic inflow with maximum velocity $2 \mathrm{~cm} / \mathrm{s}$ and Reynolds number approximately 20) carrying dissolved biocide enters the domain from the left boundary and leaves the domain from the right boundary. The velocity at the top and bottom boundaries is zero. In (1)-(3), noflux boundary conditions are assumed for the bottom and top boundaries, Dirichlet boundary conditions are assumed for the left boundary, and outflow boundary conditions (Ol'shanskii and Staroverov 2000) are assumed for the right boundary. The simulation is run for a time period of 60 minutes, corresponding to the experiment time in Davison et al. (2010). The length and time scales used in the nondimensionalization are $h_{0}=10^{-3} \mathrm{~m}$ and $t_{0}=5 \times 10^{-2} \mathrm{~s}$, thus the associated velocity scale is $h_{0} / t_{0}=0.02 \mathrm{~m} / \mathrm{s}$ and the dimensionless velocity $\tilde{\mathbf{v}}=1$ corresponding to dimensional velocity $\mathbf{v}=0.02 \mathrm{~m} / \mathrm{s}$, which is the bulk flow velocity in Davison et al. (2010). We remark that in the subsequent figures, numerical results are plotted with dimensionless spatial variables and dimensional temporal variable.

Three bacteria-killing biocides, chlorine, QAC and nisin, are considered in the simulation. The concentration is $50 \mathrm{mg} / \mathrm{l}$ for each biocide, with an additional case of $10 \mathrm{mg} / \mathrm{l}$ for chlorine (corresponding to the experiments in Davison et al. 2010). The difference between different biocides is characterized by their effective diffusion coefficient in the biofilm $D_{e}$, their effect on the effective viscosity of the biofilm $v$, and their maximum consumption rate $K_{1}$ due to reaction with the biofilm. For all biocides, $D_{e}$ is calculated according to the formula given in (7), whose value is significantly reduced within the biofilm where $\phi_{b}>0$ comparing to the diffusion coefficient $D_{0}$ in the bulk fluid. To model the fact that nisin penetrated the biofilm faster than all other biocides and killed the bacteria uniformly from all parts of the biofilm despite its smallest diffusion coefficient in water, we use a much smaller value of $K_{1}\left(2 \times 10^{-7}\right)$ than that for chlorine and QAC $\left(2 \times 10^{-4}\right)$. For nisin and QAC, the effective viscosity $v$ is calculated as the simple volume average given in (8), and for chlorine, $v$ is calculated by formula given in (9). In summary, in our model, nisin penetrates the biofilm relatively fast despite its smaller diffusion coefficient because its consumption rate due to the reaction with the biofilm is very slow; the penetration of chlorine and QAC are retarded by both the reduced diffusivity $D_{e}$ and a much faster (comparing to nisin) consumption rate due to the reaction with the biofilm; QAC and nisin do not weaken the mechanical property of the biofilm but chlorine does. 
Table 5 Biocides diffusive penetration time by numerical simulation

\begin{tabular}{lllrc}
\hline Biocide & Aqueous diffusivity $D_{d}^{0}\left(\mathrm{~m}^{2} \mathrm{~s}^{-1}\right)$ & Concentration $(\mathrm{mg} / \mathrm{l})$ & $t_{50}(\mathrm{~s})$ & $T_{50 B}(\mathrm{~min})$ \\
\hline Chlorine & $1.9 \times 10^{-9}$ & 10 & 11 & 48 \\
Chlorine & $1.9 \times 10^{-9}$ & 50 & 11 & 11 \\
QAC & $0.427 \times 10^{-9}$ & 50 & 49 & 47 \\
Nisin & $0.19 \times 10^{-9}$ & 50 & 110 & 8 \\
\hline
\end{tabular}

We quantify the killing effects of biocides in our model by the two quantities introduced in Sect. 2.3: $t_{50}$ as the time required for a biocide to attain $50 \%$ of its bulk fluid concentration at the center of the semicircular biofilm through diffusion in the absence of reaction or sorption; $T_{50 B}$ as the combined penetration and action time required for the live bacteria volume fraction $\phi_{b l}$ at the center of the semicircular biofilm to fall to $50 \%$ of its original value. We consider the biofilm with radius $R=$ $0.3 \mathrm{~mm}$ and volume fraction $\phi_{b}=0.4$, and $t_{50}$ is calculated by the formula $t_{50}=$ $0.139 R^{2} /\left(\phi_{s} D_{d}^{0}\right)$ where $\phi_{s}=1-\phi_{b}=0.6$. $T_{50 B}$ is extracted from the numerical simulation as the time when the value $\phi_{b l}$ at the center of the biofilm approximately drops to 0.2 . Table 5 gives the value of $t_{50}$ (in seconds) and $T_{50 B}$ (in minutes). The interpretation of these results is given below along with the explanation of Figs. 1-4 and also in comparison with the experimental results in Davison et al. (2010) shown in Table 2.

Figures 1-4 are the contour plots of live bacteria volume fraction $\phi_{b l}$ (left column) and dead bacteria volume fraction $\phi_{b d}$ (right column) at various time, under the treatment of $10 \mathrm{mg} / \mathrm{l}$ chlorine, $50 \mathrm{mg} / \mathrm{l}$ chlorine, $50 \mathrm{ml} / \mathrm{l} \mathrm{QAC}$ and $50 \mathrm{mg} / \mathrm{l} \mathrm{nisin}$, respectively. These figures show the spatial and temporal patterns of antimicrobial action in the biofilm.

Figure 1 shows the contour plots of $\phi_{b l}$ and $\phi_{b d}$ at $t=0,20,40,60$ min under the treatment of $10 \mathrm{mg} / \mathrm{l}$ chlorine. At $t=0$, the biofilm consists of live bacteria only. As time goes by, chlorine penetrates the biofilm through diffusion, and its antimicrobial action is localized around the periphery of the biofilm and migrates steadily inward. The live bacteria are killed in a stratified pattern, and the ones at the center of the biofilm survive for the longest time because the biocide reaches there last; see Figs. 1(a), (c), (e), (g). At $t=60 \mathrm{~min}$, the value of $\phi_{b l}$ is of order $10^{-7}$ which indicates that all live bacteria are virtually killed. The dead bacteria as shown by Figs. 1(b), (d), (f), (h). Furthermore, the effective viscosity $v$ for biofilm treated by chlorine is calculated by (9), in which the viscosity of the dead bacteria eventually approaches to that of the solvent $v_{s}$. Namely, the dead bacteria are liquefied and hence vulnerable to removal by shear forces from the surrounding bulk flow. We observe that at $t=20 \mathrm{~min}$, the dead bacteria is tilted slightly toward the downstream direction, showing the effect of shear stress from the bulk flow. But the deformation is small indicating that the dead bacteria still hold most of their mechanical strength. At $t=40 \mathrm{~min}$, with longer treatment of chlorine, the dead bacteria in the outer region of the biofilm are fully liquefied, stripped from the biofilm, and carried away by the bulk flow (leaves the computation domain at the right boundary). The removal of biofilm caused by chlorine is mainly in the form of erosion. At $t=60 \mathrm{~min}$, more than $60 \%$ 


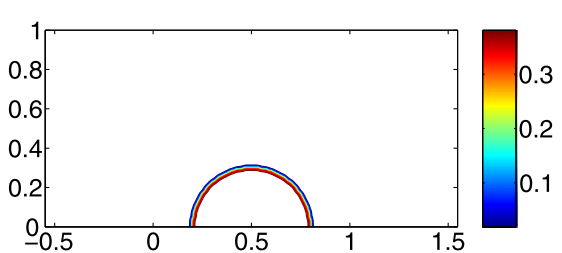

(a) $\phi_{b \mid}$ at $t=0$

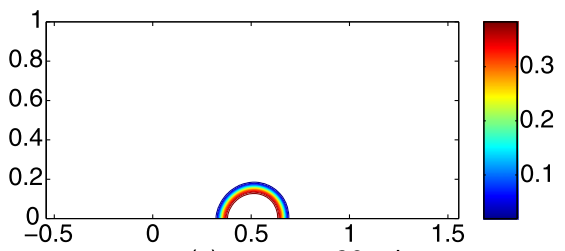

(c) $\phi_{b l}$ at $t=20 \mathrm{~min}$

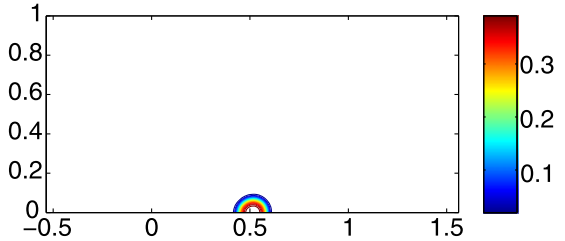

(e) $\phi_{b l}$ at $t=40 \mathrm{~min}$

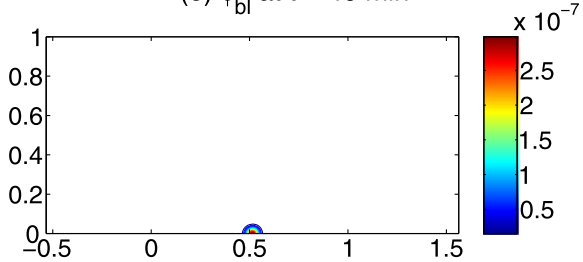

(g) $\phi_{b l}$ at $\mathrm{t}=60 \mathrm{~min}$

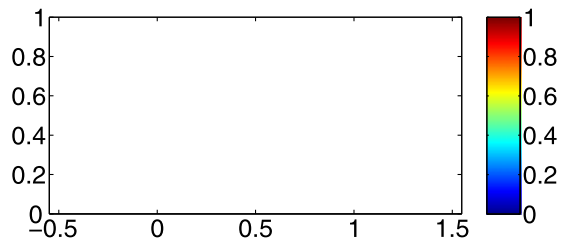

(b) $\phi_{\text {bd }}$ at $\mathrm{t}=0$

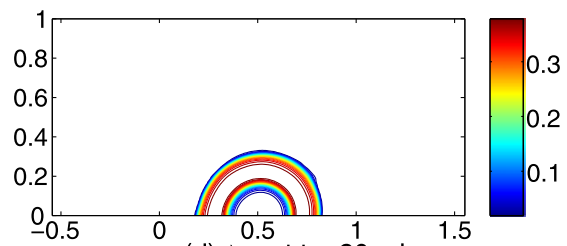

(d) $\phi_{\text {bd }}$ at $t=20 \mathrm{~min}$

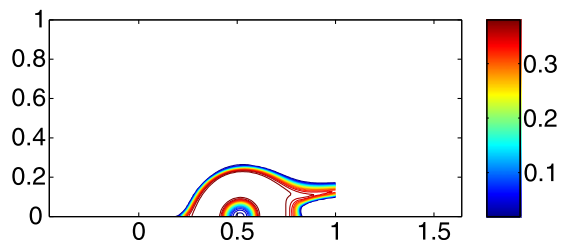

(f) $\phi_{\text {bd }}$ at $\mathrm{t}=40 \mathrm{~min}$

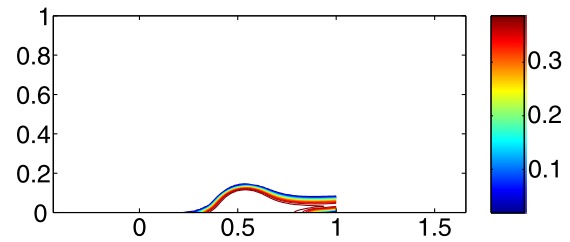

(h) $\phi_{\text {bd }}$ at $\mathrm{t}=60 \mathrm{~min}$

Fig. 1 (Color online) Contour of live and dead bacteria treated by $10 \mathrm{mg} / \mathrm{l}$ Chlorine

of the dead bacteria are removed from the biofilm; see also Fig. 7(a). Table 5 shows that the estimated diffusive penetration time in the absence of reaction $t_{50}=11 \mathrm{~s}$ is much shorter than the combined diffusion and reaction time $T_{50 \mathrm{~B}}=48 \mathrm{~min}$ extracted from the numerical simulation. This qualitatively agrees with the experimental results from Davison et al. (2010) where $t_{50}=3 \mathrm{~s}$ and $T_{50 \mathrm{~B}}=46 \pm 33 \mathrm{~min}$ and indicates that formula (7) describes the diffusion of chlorine in the biofilm appropriately. The discrepancy in $t_{50}$ is mainly due to that the radius $R$ is $0.3 \mathrm{~mm}$ in our estimate and $0.15 \mathrm{~mm}$ in Davison et al. (2010). The discrepancy in $T_{50 \mathrm{~B}}$ is also expected since the experimental value is obtained by averaging results of several runs, and the size of the biofilm clusters in experiment generally differs from the value used in our numerical simulation. We remark that in our model the live bacteria maintain their original viscosity $v_{b}$, therefore, their deformation is very small within the simulation time (60 min) due to the large viscosity $\left(v_{b} \gg v_{s}\right)$.

Figure 2 shows the contour plots of $\phi_{b l}$ and $\phi_{b d}$ at $t=0,10,15,20$ min under the treatment of $50 \mathrm{mg} / \mathrm{l}$ chlorine. The pattern of killing of live bacteria and removal of biofilm are similar to those of $10 \mathrm{mg} / \mathrm{l}$ chlorine treatment, except it happens in a faster 

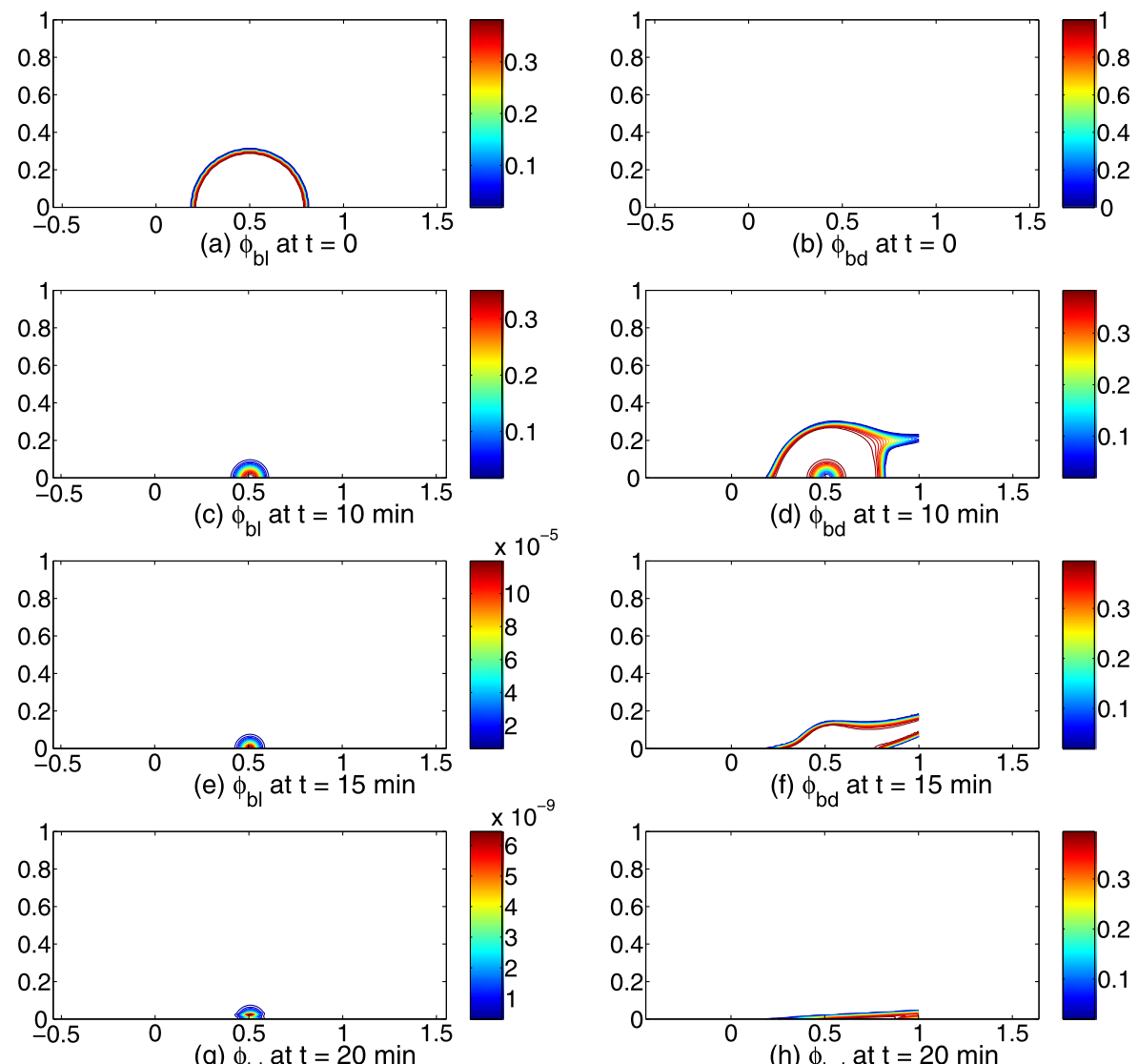

(d) $\phi_{b d}$ at $t=10 \mathrm{~min}$
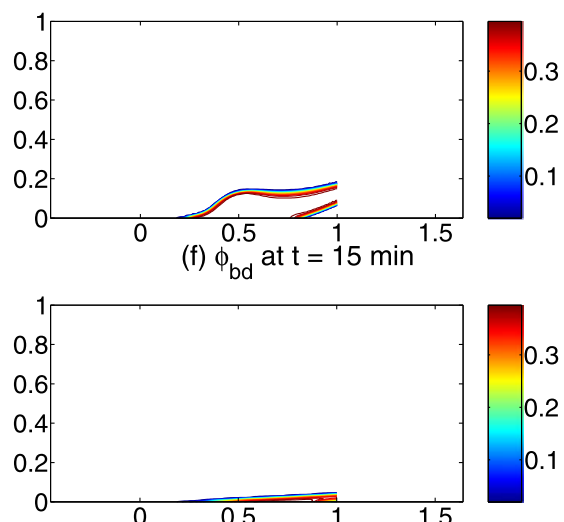

(h) $\phi_{\text {bd }}$ at $\mathrm{t}=20 \mathrm{~min}$

Fig. 2 (Color online) Contour of live and dead bacteria treated by $50 \mathrm{mg} / \mathrm{l} \mathrm{Chlorine}$

pace due to the higher concentration of chlorine. We observe $\phi_{b l}$ is of order $10^{-5}$ at $t=15 \mathrm{~min}$ and almost all biofilm are removed at $t=20 \mathrm{~min}$. Again, the numerical simulation gives $t_{50}=11 \mathrm{~s}$ and $T_{50 B}=11 \mathrm{~min}$ which agree qualitatively with the experimental values $t_{50}=3 \mathrm{~s}$ and $T_{50 B}=21 \pm 16 \mathrm{~min}$.

Figure 3 shows the contour plots of $\phi_{b l}$ and $\phi_{b d}$ at $t=0,20,40,60$ min under the treatment of $50 \mathrm{mg} / \mathrm{l}$ QAC. The antimicrobial action is again localized around the periphery of the biofilm and migrates steadily inward. Since the dead bacteria maintain their original viscosity $v_{b}$, the biofilm is only stretched slightly toward the downstream direction, and no removal of biofilm occurs. The maximum value of $\phi_{b l}$ at $t=60 \mathrm{~min}$ is still around its initial value 0.4 , which indicates that the killing rate of live bacteria by $50 \mathrm{mg} / \mathrm{l}$ QAC is even slower than that of $10 \mathrm{mg} / \mathrm{l}$ chlorine. This slower killing rate could be explained by two reasons. First, the aqueous diffusion coefficient of QAC $\left(0.427 \times 10^{-9} \mathrm{~m}^{2} \mathrm{~s}^{-1}\right)$ is about 4.5 times smaller than that of chlorine $\left(1.9 \times 10^{-9} \mathrm{~m}^{2} \mathrm{~s}^{-1}\right)$; second, the dead bacteria in the outer region are not removed and protect the live bacteria inside against biocide action by reducing its effective diffusivity $D_{e}$ in the biofilm according to formula (7). For QAC, the numer- 


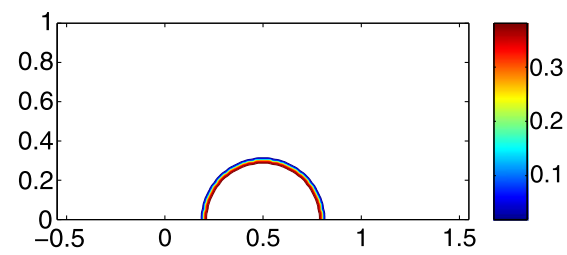

(a) $\phi_{b l}$ at $t=0$

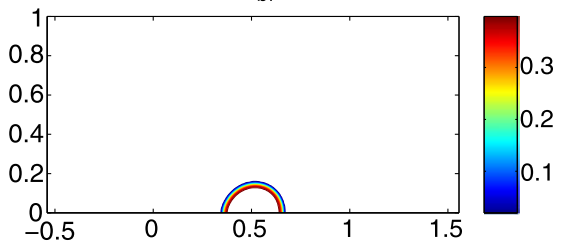

(c) $\phi_{b \mid}$ at $t=20 \mathrm{~min}$

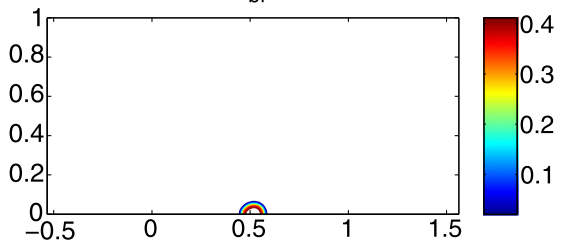

(e) $\phi_{b l}$ at $t=40 \mathrm{~min}$

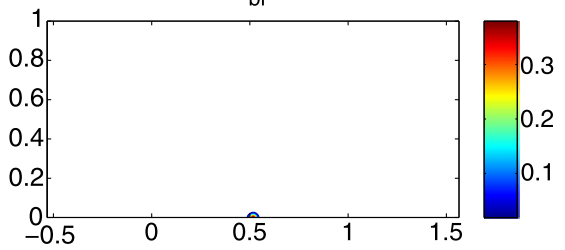

(g) $\phi_{b \mid}$ at $t=60 \mathrm{~min}$

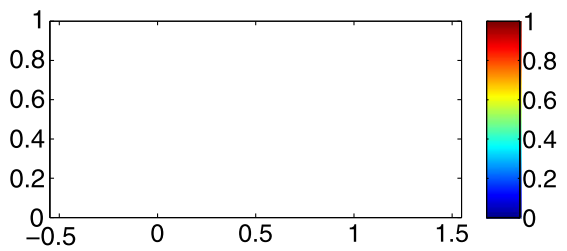

(b) $\phi_{b d}$ at $t=0$

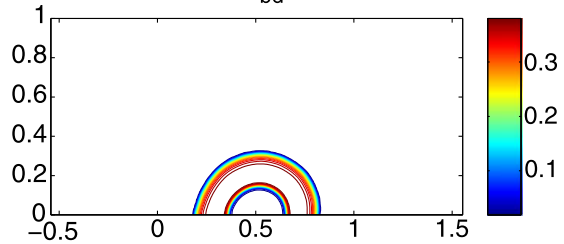

(d) $\phi_{\text {bd }}$ at t $=20 \mathrm{~min}$

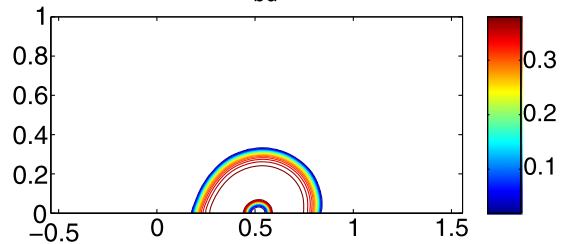

(f) $\phi_{\text {bd }}$ at t $=40 \mathrm{~min}$

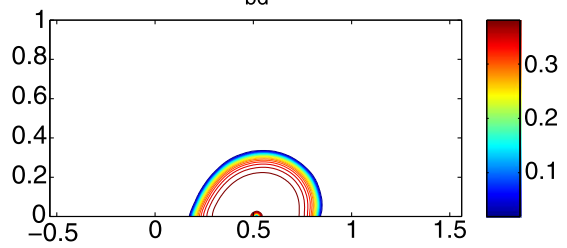

(h) $\phi_{b d}$ at t $=60 \mathrm{~min}$

Fig. 3 (Color online) Contour of live and dead bacteria treated by $50 \mathrm{mg} / \mathrm{l}$ QAC

ical simulation gives $t_{50}=49 \mathrm{~s}$ and $T_{50 B}=47$ min which agree qualitatively with the experimental values $t_{50}=24 \mathrm{~s}$ and $T_{50 B}=25 \pm 15 \mathrm{~min}$.

Figure 4 shows the contour plots of $\phi_{b l}$ and $\phi_{b d}$ at $t=0,5,10,20$ min under the treatment of $50 \mathrm{mg} / \mathrm{l}$ nisin. Since the value of $K_{1}$ for nisin $\left(2 \times 10^{-7}\right)$ is much smaller than that for chlorine and QAC $\left(2 \times 10^{-4}\right)$, nisin is almost not consumed as it penetrates the biofilm. As a result, nisin is among the fastest killing biocides (comparable to $50 \mathrm{mg} / \mathrm{l}$ chlorine, and much faster than $50 \mathrm{mg} / \mathrm{l}$ QAC and $10 \mathrm{mg} / \mathrm{l}$ chlorine), and it kills the bacteria almost uniformly from all parts of the biofilm, despite its smallest aqueous diffusion coefficient $\left(0.19 \times 10^{-9} \mathrm{~m}^{2} \mathrm{~s}^{-1}\right)$ among all biocides considered. $\phi_{b l}$ is of order $10^{-3}$ at $t=20 \mathrm{~min}$. Also, due to its uniform killing effect in the biofilm, the action of nisin is not as sharply stratified as in the cases of chlorine and QAC, and the interface between live and dead bacteria is more smeared out. Furthermore, the discrepancy between $t_{50}=110 \mathrm{~s}$ and $T_{50 \mathrm{~B}}=8 \mathrm{~min}$ for nisin is much smaller than that of chlorine and QAC, which agree qualitatively with the experimental values $t_{50}=55 \mathrm{~s}$ and $T_{50 \mathrm{~B}}=4 \pm 1 \mathrm{~min}$. 


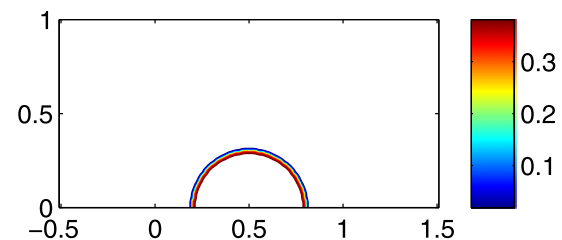

(a) $\phi_{b l}$ at $t=0$

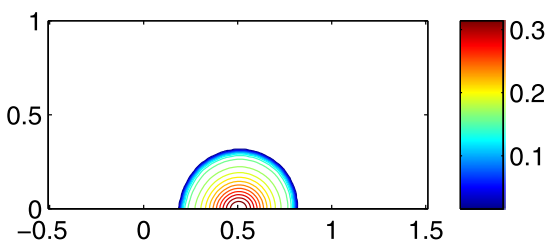

(c) $\phi_{b l}$ at t $=5 \mathrm{~min}$

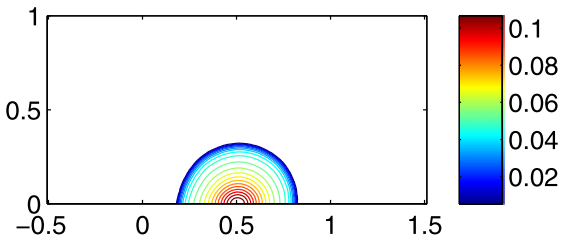

(e) $\phi_{b l}$ at $t=10 \mathrm{~min}$

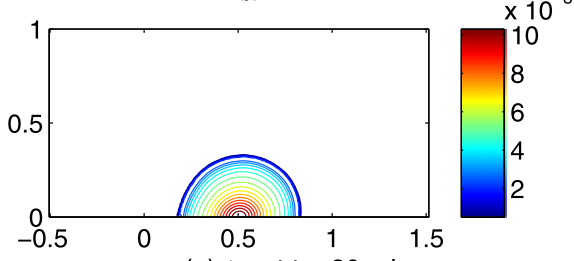

(g) $\phi_{b l}$ at t $=20 \mathrm{~min}$

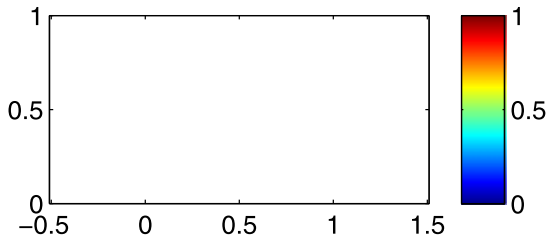

(b) $\phi_{b d}$ at $t=0$

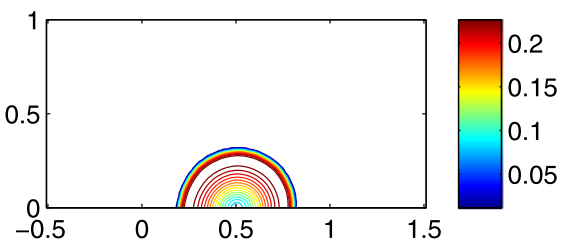

(d) $\phi_{\text {bd }}$ at t $=5 \mathrm{~min}$

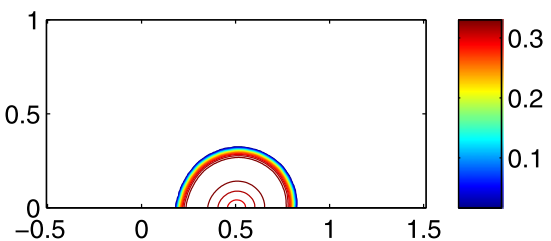

(f) $\phi_{b d}$ at t $=10 \mathrm{~min}$

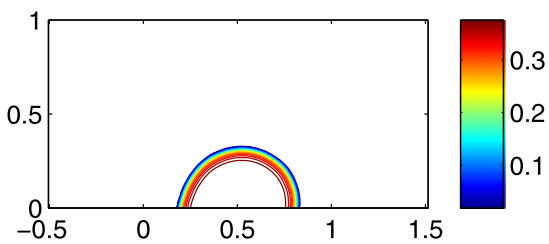

(h) $\phi_{b d}$ at $t=20 \mathrm{~min}$

Fig. 4 (Color online) Contour of live and dead bacteria treated by $50 \mathrm{mg} / \mathrm{l} \mathrm{Nisin}$

To aid the interpretation of the numerical results, Fig. 5 gives the plots of relative live bacteria intensity versus time at three different regions in the biofilm for all biocide treatments. The initial semicircular biofilm with radius $R=0.3 \mathrm{~mm}$ is divided into three regions consisting of the periphery $\Omega_{p}(2 / 3 R \leq r \leq R)$, the medial region $\Omega_{m}(1 / 3 R \leq r<2 / 3 R)$ and the center $\Omega_{c}(r<2 / 3 R)$ where $r$ is the distance from the center of the biofilm. Each plot corresponds to a particular biocide treatment, where the horizontal axis is time in minutes, and the vertical axis is the integral of $\phi_{b l}$ in a particular region divided by its initial value in the same region. Namely, the three plotted functions are

$$
\frac{\int_{\Omega_{p}} \phi_{b l}(x, t) d x}{\Omega_{p} \phi_{b l}(x, 0) d x}, \quad \frac{\int_{\Omega_{m}} \phi_{b l}(x, t) d x}{\Omega_{m} \phi_{b l}(x, 0) d x}, \quad \frac{\int_{\Omega_{c}} \phi_{b l}(x, t) d x}{\Omega_{c} \phi_{b l}(x, 0) d x} .
$$

We observe that the antimicrobial action of each biocide is localized around the periphery and migrates steadily inward. $50 \mathrm{mg} / \mathrm{l}$ nisin has the fastest killing rate, fol- 


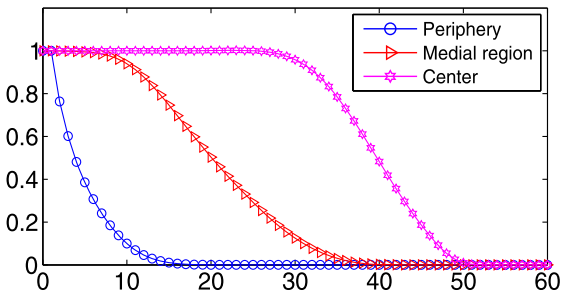

(a) Chlorine $-10 \mathrm{mg} / \mathrm{l}$

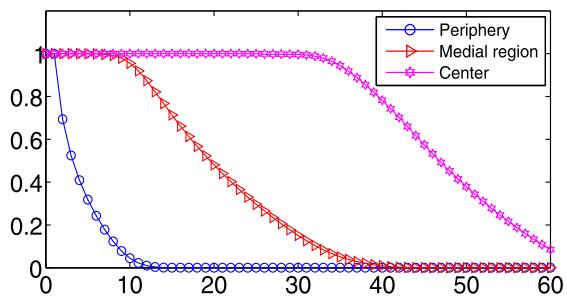

(c) QAC - $50 \mathrm{mg} / \mathrm{l}$

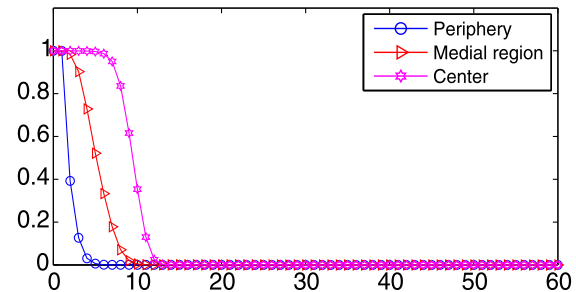

(b) Chlorine $-50 \mathrm{mg} / \mathrm{l}$

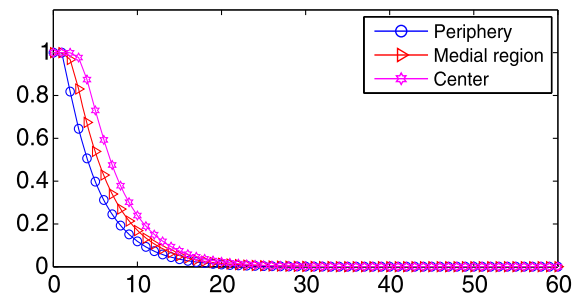

(d) Nisin - $50 \mathrm{mg} / \mathrm{l}$

Fig. 5 The relative intensity of $\phi_{b l}$ at the peripheral, medial and central regions

lowed by $50 \mathrm{mg} / \mathrm{l}$ chlorine, $10 \mathrm{mg} / \mathrm{l}$ chlorine and $50 \mathrm{mg} / \mathrm{l} \mathrm{QAC}$, in decreasing order, which is consistent with results from Figs. 1-4. We remark that since the live bacteria maintain their original viscosity $v_{b}$ and their deformation due to shear forces from the bulk flow is very small within the simulation time, the convection of $\phi_{b l}$ is negligible and the change of $\phi_{b l}$ in each of the three regions is mainly due to the effect of biocide. Therefore, quantities defined in (12) appropriately describes the antimicrobial action of the biocides. Figure 6 shows the corresponding experimental results from Davison et al. (2010) where the relative intensity plotted on the $y$ axis is the fluorescence intensity at a particular region of the biofilm divided by the initial intensity in that same region. Shown are measurements made near the periphery of the cell cluster (black symbols), a medial location of the cell cluster (open symbols), and the center of the cell cluster (gray symbols). We can see our numerical simulation agrees qualitatively with the experimental results.

Figure 7(a) gives the plot of total bacteria (live and dead) left in the computation domain $\Omega$ normalized by its initial value versus time under the treatment of chlorine. Namely, the function plotted is

$$
\frac{\int_{\Omega} \phi_{b}(x, t) d x}{{ }_{\Omega} \phi_{b}(x, 0) d x} .
$$

We observe under $10 \mathrm{mg} / \mathrm{l}$ chlorine, about $68 \%$ biofilm is removed after $60 \mathrm{~min}$, and under $50 \mathrm{mg} / \mathrm{l}$ chlorine, the biofilm is almost completely removed after $30 \mathrm{~min}$. Figure 7(b) shows the corresponding experimental results from Davison et al. (2010) where erosion of biofilm clusters, as indicated by reduction in cluster area, during treatment with PBS (control), $10 \mathrm{mg} / \mathrm{l}$ chlorine, or $50 \mathrm{mg} / \mathrm{l}$ chlorine is given. Different curves in Fig. 7(b) correspond to different runs of the experiment. We again see our numerical simulation agrees qualitatively with one of the experimental runs. The cases for QAC and nisin are not shown since they do not cause biofilm removal. 

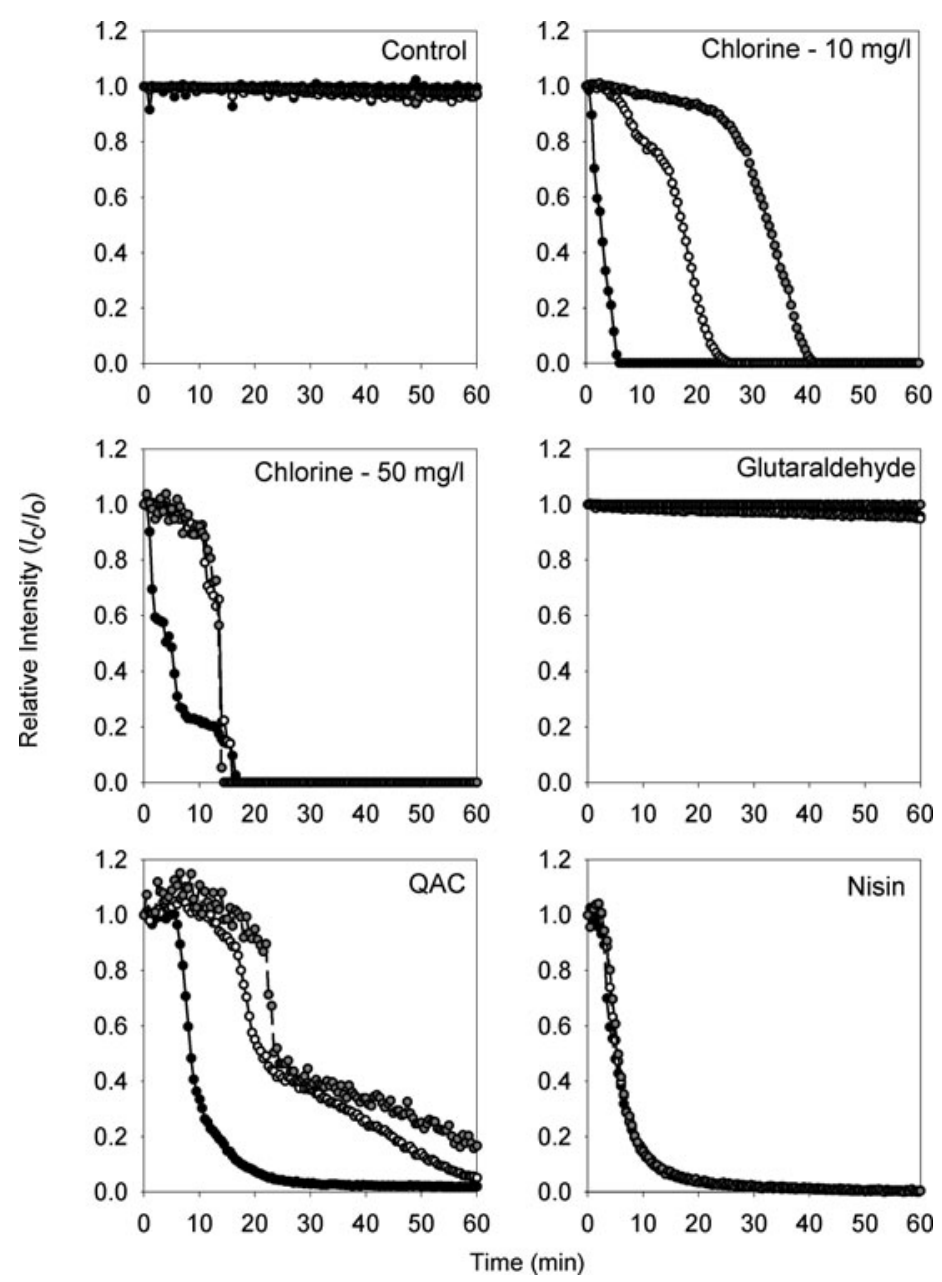

Fig. 6 Image analysis of fluorescence loss in CAM-stained S. epidermidis biofilms treated under continuous flow as indicated in each panel. Reprinted by permission of American Society for Microbiology from Davison et al. (2010)

\section{Conclusion}

We have applied a mixture model to study the antimicrobial action of biocide against biofilm. By incorporating appropriate formula for the effective diffusion coefficient of the biocide in the biofilm and effective viscosity of the biofilm, as well as different consumption rates for different biocides, the model describes the effect of the biocide on both killing the bacteria and weakening its mechanical property. The NavierStokes equation is used to model the flow, and removal of the biofilm due to the shear stress of the bulk flow is captured. The numerical simulation of our model produces results that qualitatively agree with available experimental data. Among the three biocides considered, nisin is perhaps the most effective one in terms of killing effect, but chlorine is the only biocide which weakens the biofilm's mechanical properties 
Fig. 7 Comparison of numerical and experimental results of biomass left under treatment of chlorine.

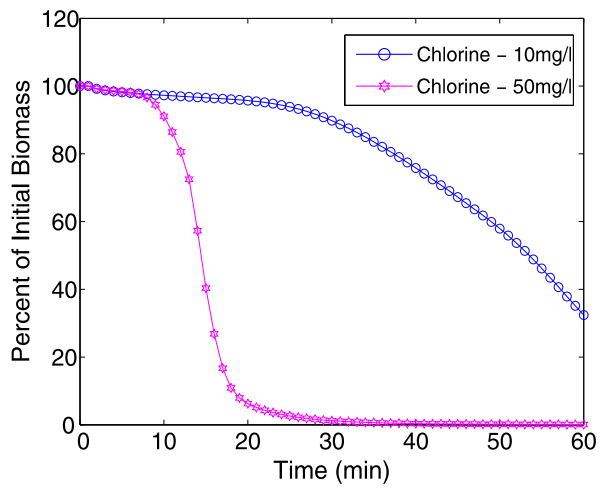

(a) Numerical results of total biomass left for biofilm treated by chlorine.
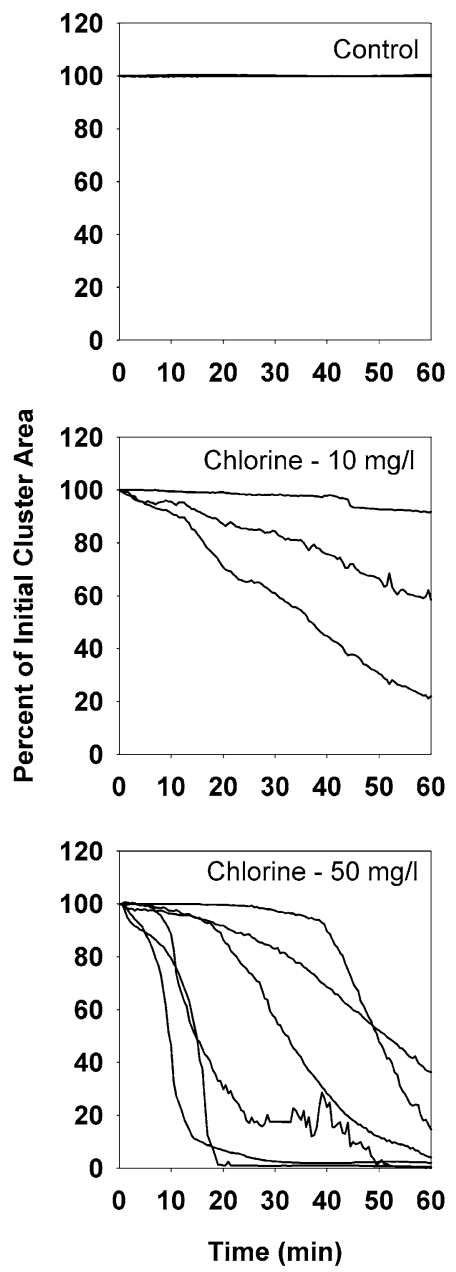

(b) Reprinted by permission of American Society for Microbiology from [7]. 
and subsequently causes biofilm removal under bulk flow. These results reinforce the distinction between killing bacteria in the biofilm and biofilm removal, and chlorine is a better choice if biofilm removal is the ultimate goal. Our model provides a framework for modeling experiments of biocide action against biofilms where both viability reduction and the hydraulic contribution to the removal of biofilm weakened by reaction with biocide are important. Furthermore, by adding a new variable for nutrient concentration and modifying the reaction kinetics accordingly, the model can be extended to describe processes where both biofilm growth and disinfection are important.

Acknowledgements T. Zhang is supported by NSF through Grant DMS-0934696. The author thanks Philip Stewart for many helpful suggestions and comments.

\section{References}

Anguige, K., King, J. R., \& Ward, J. P. (2005). Modelling antibiotic- and anti-quorum sensing treatment of a spatially-structured pseudomonas aeruginosa population. J. Math. Biol., 51, 557-594.

Chambless, J. D., Hunt, S. M., \& Stewart, P. S. (2006). A three-dimensional computer model of four hypothetical mechanisms protecting biofilms from antimicrobials. Appl. Environ. Microbiol., 72, 20052013.

Characklis, W. G., \& Marshall, K. C. (1990). Biofilms. New York: Wiley.

Cogan, N. G. (2008). Two-fluid model of biofilm disinfection. Bull. Math. Biol., 70, 800-819.

Cogan, N. G., Cortez, R., \& Fauci, L. (2005). Modeling physiological resistance in bacterial biofilms. Bull. Math. Biol., 67, 831-853.

Costerton, J. W., Lewandowski, Z., Caldwell, D. E., Korber, D. R., \& Lappin-Scott, H. M. (1995). Microbial biofilms. Annu. Rev. Microbiol., 49, 711-745.

Davison, W. M., Pitts, B., \& Stewart, P. S. (2010). Spatial and temporal patterns of biocide action against staphylococcus epidermidis biofilms. Antimicrob. Agents Chemother., 54, 2920-2927.

Demaret, L., Eberl, H. J., Efendiev, M. A., \& Lasser, R. (2008). Analysis and simulation of a meso-scale model of diffusive resistance of bacterial biofilms to penetration of antibiotics. Adv. Math. Sci. Appl., 18, 269-304.

Dodds, M. G., Grobe, K. J., \& Stewart, P. S. (2000). Modeling biofilm antimicrobial resistance. Biotechnol. Bioeng., 68, 456-465.

Doi, M. (1995). Introduction to polymer physics. Oxford: Oxford Science Publications.

Eberl, H. J., \& Sudarsan, R. (2008). Exposure of biofilms to slow flow fields: The convective contribution to growth and disinfection. J. Theor. Biol., 253, 788-807.

Gibbons, R. J., \& van Houte, J. (1980). Bacterial adherence and the formation of dental plaque. In E. M. Beachey (Ed.), Bacterial adherence (pp. 61-104). London: Chapman \& Hall.

Guermond, J. L., Minev, P., \& Shen, J. (2006). An overview of projection methods for incompressible flows. Comput. Methods Appl. Mech. Eng., 95, 6011-6045.

Hinson, R. K., \& Kocher, W. M. (1996). Model for effective diffusivities in aerobic biofilms. J. Environ. Eng., 122(11), 1023-1030.

Jesaitis, A. J., Franklin, M. J., Berglund, D., Sasaki, M., Lord, C. I., Bleazard, J. B., Duffy, J. E., Beyenal, H., \& Lewandowski, Z. (2003). Compromised host defense on pseudomonas aeruginosa biofilms: characterization of neutrophil and biofilm interactions. J. Immunol., 171, 4329-4339.

Klapper, I., \& Dockery, J. (2010). Mathematical description of microbial biofilms. SIAM Rev., 52, 221265.

Lewis, K. (2001). Riddle of biofilm resistance. Antimicrob. Agents Chemother, 45, 999-1007.

Lindley, B., Wang, Q., \& Zhang, T. (2011). A multicomponent model for biofilm-drug interaction. Discrete Contin. Dyn. Syst., Ser. B, 15, 417-456.

Ol'shanskii, M. A., \& Staroverov, V. M. (2000). On simulation of outflow boundary conditions in finite difference calculations for incompressible fluid. Int. J. Numer. Methods Fluids, 33, 499-534.

Passerini, L., Lam, K., Costerton, J. W., \& King, E. G. (1992). Biofilms on indwelling vascular catheters. Clin. Crit. Care Med., 20, 665-673. 
Saad, Y., \& Schultz, M. H. (1986). Gmres: A generalized minimal residual algorithm for solving nonsymmetric linear systems. SIAM J. Sci. Stat. Comput., 7, 856-869.

Sanderson, S. S., \& Stewart, P. S. (1997). Evidence of bacterial adaptation to monochloramine in pseudomonas aeruginosa biofilms and evaluation of biocide action model. Biotechnol. Bioeng., 56, 201209.

Schein, O. D., Glynn, R. J., Seddon, J. M., \& Kenyon, K. R. (1989). The relative risk of ulcerative keratitis among users of daily-wear and extended-wear soft contact lenses. N. Engl. J. Med., 321, 773-778.

Shaw, T., Winston, M., Rupp, C. J., Klapper, I., \& Stoodley, P. (2004). Commonality of elastic relaxation times in biofilms. Phys. Rev. Lett., 93, 098102.

Stewart, P.S. (1996). Theoretical aspects of antibiotic diffusion into microbial biofilms. Antimicrob. Agents Chemother, 40, 2517-2522.

Stewart, P. S. (2002). Mechanisms of antibiotic resistance in bacterial biofilms. Int. J. Med. Microbiol., 292, 107-113.

Stewart, P. S. (2003). Diffusion in biofilms. J. Bacteriol., 185, 1485-1491.

Stewart, P. S., \& Costerton, J. W. (2001). Antibiotic resistance of bacteria in biofilms. Lancet, 358, 135138.

Stickler, D., Ganderton, L., King, J., Nettleton, J., \& Winters, C. (1993). Proteus mirabilis biofilms and the encrustation of urethral catheters. Urol. Res., 21(6), 407-411.

Szomolay, B., Klapper, I., Dockery, J., \& Stewart, P. S. (2005). Adaptive responses to antimicrobial agents in biofilms. Environ. Microbiol., 7, 1186-1191.

Zhang, T., \& Klapper, I. (2010). Mathematical model of biofilm induced calcite precipitation. Water Sci. Technol., 61, 2957-2964.

Zhang, T., Cogan, N., \& Wang, Q. (2008a). Phase-field models for biofilms I. Theory and simulations. SIAM J. Appl. Math., 69, 641-669.

Zhang, T., Cogan, N., \& Wang, Q. (2008b). Phase-field models for biofilms. II. 2-D numerical simulations of biofilm-flow interaction. Commun. Comput. Phys., 4, 72-101. 